\title{
AVALIAÇÃO DE FERRAMENTAS DE TECNOLOGIA DA INFORMAÇÃO NA CONSTRUÇÃO COM FUNCIONALIDADES MÓVEIS COMPATÍVEIS AOS ITENS DA NBR ISO 9001:2008
}

\section{ASSESSMENT TOOL FOR INFORMATION IN CONSTRUCTION TECHNOLOGY WITH MOBILE DEVICES COMPATIBLE WITH ITEMS OF NBR ISO 9001:2008}

\author{
Romeu da S. Neiva Neto; Regina C. Ruschel²; Flávio A. Picchi ${ }^{3}$
}

Recebido em 11 de dezembro de 2012; recebido para revisão em 14 de dezembro de 2012; aceito em 30 de dezembro de 2012; disponível on-line em 10 de janeiro de 2013.

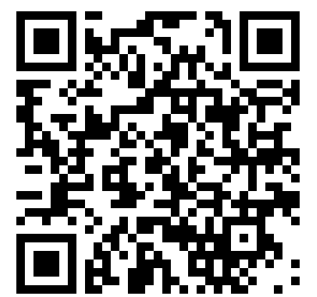

\section{PALAVRAS CHAVES:}

Gestão da Qualidade;

Tecnologia da Informação na Construção (TIC);

NBR ISO 9001.

\section{KEYWORDS:}

Quality Management; Information Technology in Construction (ICT);

NBR ISO 9001.

\begin{abstract}
RESUMO: A certificação da norma NBR/ISO 9001:2008 se tornou uma iniciativa comum dentro do setor da construção civil, visando a competitividade dessas empresas. O presente trabalho tem como objetivo identificar as funcionalidades de softwares destinados a gestão da qualidade compatíveis com dispositivos móveis e sua aplicabilidade como facilitadoras de práticas, para mostrar como a implantação de ferramentas de Tecnologia da Informação e Comunicação (TIC), juntamente com a aceleração da comunicação na sociedade imposta pela mobilidade pode gerar uma maior trabalhabilidade nos processos da organização, agilizando a burocracia $e$ minimizando a produção de papel na certificação citada anteriormente. A pesquisa desenvolvese como um estudo de caso. A primeira etapa do estudo consistiu em entrevistas feitas com representantes de dois softwares do mercado da construção a respeito das funcionalidades dos softwares alinhadas aos conceitos da norma NBR ISO 9001:2008. A segunda etapa do estudo consistiu no desenvolvimento de um Quadro comparativa para mapear a adequação das funcionalidades de softwares ao Sistema de Gestão da Qualidade. Desenvolve-se então um cenário de incorporação e mapeamento de recursos de mobilidade na implantação de SGQ e discute-se melhorias resultantes ao processo de qualidade. Os dados coletados servirão de parâmetros para evidenciar exemplos de aplicação de mobilidade sem que esses processos fujam do escopo das normativas da ISO para emprego em empresas do setor da construção civil.
\end{abstract}

\begin{abstract}
Certification of NBR / ISO 9001:2008 has become a common initiative within the construction industry, aiming their competitiveness. The present work aims to identify the features of software for quality management compatible with mobile devices and their applicability as facilitating practices, to show how the deployment tools of Information and Communication Technology (ICT), together with the acceleration of communication in society imposed by mobility can generate greater workability in the processes of the organizations, streamlining the bureaucracy and minimizing the production of paper in the certification mentioned above. The research was developed as a case study. The first stage of the study consisted of interviews with representatives of two software of the construction market regarding the functionality of the software aligned to the concepts of ISO 9001:2008. The second stage of the study consisted of developing a comparative table to map the suitability of the software features to the Quality Management System. Then develops a scenario incorporating mapping and mobility features in the implementation of QMS and discusses improvements to the quality process. The data collected will serve as a parameter to highlight examples of application of mobility without these processes escape the scope of the ISO standards for use in companies of the construction industry.
\end{abstract}

\footnotetext{
* Contato com os autores:

1e-mail : romeuneiva@gmail.com (R. da S. Neiva Neto)

Eng. Civil Mestrando do Curso de Engenharia Civil da Faculdade de Engenharia Civil, Arquitetura e Urbanismo (FEC/UNICAMP)

2e-mail : ruschel@fec.unicamp.br (R. C. Ruschel)

Professora Dra. da Faculdade de Engenharia Civil, Arquitetura e Urbanismo (FEC/UNICAMP)

${ }^{3}$ e-mail : fpicchi@lean.org.br (F. A. Picchi)

Professor Dr. da Faculdade de Engenharia Civil, Arquitetura e Urbanismo (FEC/UNICAMP)
} 


\section{INTRODUÇÃO}

Um dos fatores mais relevantes na discussão sobre tecnologia da informação e comunicação recentes é a questão da mobilidade da informação. Os processos corporativos vêm sofrendo um fenômeno particular com o desenvolvimento dos smartphones e o surgimento de tablets para auxílio dos mesmos. Porém, o mercado ainda espera resultados maiores que compensem o tempo e custo que envolva a adoção dessas tecnologias em seus processos existentes (CROCKER, 2011; GIBBS, 2011; WOLF, 2012).

A ideia de computadores portáteis, com telas touchscreen que possibilita a mobilidade da informação já existe há alguns anos, porém apenas há pouco tempo com o sucesso dos tablets, criou-se um mercado competitivo de dispositivos com custos e acessibilidades viáveis aos processos corporativos de forma mais incisiva. Isso impulsionou vários setores da economia a investirem no desenvolvimento de aplicativos próprios para seus setores passarem a utilizar tablets para os mais variados serviços. Estes aplicativos são desde apresentações de informações de venda de produtos contendo informações interativas à possibilidade de videoconferências com equipes de produção alocadas em um canteiro de obra (GIBBS, 2011).

Tornar a mobilidade um movimento impulsionador da economia está na forma de relacionála a infraestrutura de telecomunicação (KOUTROUMPIS, 2009). No Brasil essa infraestrutura passou por transformações profundas a partir de 1997 com a privatização das Telecomunicações Brasileiras S/A (Telebrás) com a Lei Geral de Telecomunicações (LGT) (Lei no 9.472, de 16 de julho de 1997). Isso trouxe uma dinâmica ao setor que, juntamente com os avanços tecnológicos, possibilitou a expansão acelerada das infraestruturas de telecomunicação, incluindo os serviços de telefonia fixa, móvel, internet, televisão e mais recentemente a infraestrutura de redes de terceira geração (3G) (IPEA, 2010). Tais melhorias trouxeram o acesso à tecnologias de informação e comunicação a grande maioria da população brasileira. Ou seja, com mercados consumidores tendo maior acesso a informação, a economia se expandiu em um ritmo muito mais acelerado (GARCIA, 1995).

O setor da construção civil em especial vem sofrendo uma pressão constante do mercado consumidor de alcançar os resultados esperados e a satisfação do cliente. O avanço em tais tecnologias móveis vem como uma forte ferramenta para mudar as formas de comunicação nesse setor. Algumas tecnologias já adotadas no setor evidenciam melhorias na construção civil nos quesitos de prazo de obra, custos de construção, redução no número de defeitos, redução do número de acidentes do trabalho, aumento da previsibilidade para tomada de decisão, aumento de produtividades e redução nos custos de operação e manutenção (BOWDEN et al., 2006).

A construção civil possui diversos processos que exigem a comunicação intensa entre as partes envolvidas. A norma NBR/ISO 9001:2008 é constantemente questionada no sentido de gerar burocratização para a troca de informação entre os agentes. O maior argumento que sustenta tais questionamentos está na questão do grande número necessário de documentos para o controle da qualidade do produto. Assim sendo, a utilização de dispositivos móveis no auxílio da coleta e registro dessas informações, vêm como facilitador e agregador de valor na maneira de se tratar tal problema.

\section{OBJETIVO}

Este trabalho tem por objetivo identificar funcionalidades de ferramentas de Tecnologia da Informação e Comunicação para a construção civil especificamente no que se refere a tecnologia móveis, e sua aplicabilidade como facilitadoras de práticas de controle decorrentes de exigências de itens da norma NBR/ISO 9001:2008.

Como método será utilizado estudo de caso exploratório, analisando-se dois softwares disponíveis no mercado brasileiro.

\section{REVISÃO BIBLIOGRÁFICA}

\subsection{EVOLUÇÃO DAS TECNOLOGIAS MÓVEIS}

Segundo Crocker (2011) por volta de $85 \%$ da população mundial atual possui telefones celulares. Enquanto o mercado de smartphones também vem crescendo constantemente com a diminuição dos preços de tais dispositivos ao redor do mundo e a competitividade vem garantindo dispositivos cada vez mais acessíveis e amigáveis aos usuários. Espera-se que o mercado de dispositivos móveis venha a crescer até $8 \%$ até o ano de 2015.

Segundo uma pesquisa apresentada por Wolf (2012), 4 em cada 5 empresas de tecnologia da informação atuais nos Estados Unidos dão suporte a tecnologias voltadas para dispositivos móveis. Podemos concluir assim que as empresas serão tomadas por 
processos auxiliados por dispositivos móveis nos próximos anos.

Existem exemplos de publicações no meio acadêmico nacional e internacional que nos mostram também tais dados se tornando realidade no mercado de diversas áreas. Alguns exemplos são os da área de saúde em que as ferramentas móveis auxiliam a coleta de dados de pacientes em programas de Atenção Básica domiciliar (CONCEIÇÃO et al., 2010). A coleta de dados sobre Epidemiologia Bucal para saúde da população com dispositivos PDA's (BREGA et al., 2008). Na área de transportes existem atividades no desenvolvimento de ferramentas móveis para coleta de dados de uma empresa de transportes (SCHAEFER, 2004). Outra área que já explora as vantagens das tecnologias móveis é a área da educação em que existem diversos exemplos de aplicações sendo utilizadas no auxilio ao ensino (ISMAIL, 2011; SIBALDO et al., 2006; VAVOULA et al., 2004). O campo da administração também possui pesquisas na área (FÜHR, 2007). Além da própria área de tecnologia de informação que está em constante desenvolvimento de novas formas de gerar aplicativos móveis que disponibilizem as mais diversas formas de interação com os usuários através das muitas plataformas e linguagens de desenvolvimento existentes (GOMES, 2005; MIGLINSKI, 2003). Porém, para que exista a proliferação dessa tecnologia de forma incisiva, é necessária uma infraestrutura de telecomunicação que de suporte a essa rede de aplicações.

\subsection{FERRAMENTAS DE SISTEMA DE GESTÃO INFORMATIZADOS PARA A CONSTRUÇÃO CIVIL BRASILEIRA}

Segundo Nascimento et al (2002) existem barreiras ligadas a diversos fatores que impedem as construtoras de investir mais incisivamente em tecnologia da informação e comunicação (TIC). Elas são barreiras ligadas aos profissionais do setor que não possuem embasamento na área na sua formação, ligadas aos processos do setor que possui uma latência na adoção de metodologias de gestão em relação a outros setores e ligadas às próprias tecnologias que ainda carecem de aprofundamento dentro da área em específico.

Dentre essas, as barreiras ligadas aos processos e a tecnologia são o foco da metodologia desse trabalho, pois houve um crescimento de investimentos nos sistemas de gestão da qualidade entre as construtoras nos últimos anos e até então o setor carecia de maneiras eficientes de otimizar seus processos. Porém, as inovações tecnológicas passaram a existir dentre as opções de gerenciamento e controle da informação para aqueles mais interessados dentro do mercado (SOUZA; AMORIN, 2001).

Os softwares de auxilio a gestão da produção encontrados no mercado são conhecidos pela sigla ERP (Enterprise Resource Planing) que segundo Peyrel (2007) caracterizam-se como sistemas de informação que pretendem suportar todas as necessidades de tomada de decisão da empresa. Esses softwares já são bem difundidos entre as empresas do mercado da construção civil brasileiro atualmente. Entretanto, devido a competitividade crescente no setor, surgem novos softwares, atendendo a novas necessidades das empresas. Esses softwares atuam mais na Gestão Operacional da Informação (GOI) e como pretende o presente trabalho, possuem funcionalidades móveis (NEGÓCIOS E EMPREENDIMENTOS, 2012).

Ferramentas de TIC para gerenciamento e controle da produção no país possuem origem de pesquisa na região Sul e Sudeste do país através de Projetos fomentados pela parceria do setor privado e órgãos de pesquisa desde o início dos anos 2000. Tais projetos envolvem instituições como a UFPR (Universidade Federal do Paraná), UFF (Universidade Federal Fluminense), UFRJ (Universidade Federal do Rio de Janeiro, CNPq entre outras empresas e órgãos fomentadores que possibilitaram o desenvolvimento de ferramentas pioneiras como o SIGMO (Sistema Integrado de Gerenciamento Móvel em Obras) (SOUZA; AMORIN, 2001) e o Plantracker (PEYERL, 2007; ZEN, 2006). Atualmente alguns softwares presentes no mercado com tais característica que podemos citar são:

1. Colaborativo, da Construtivo, sediado em São Paulo-SP

2. Mobuss, da Teclógica, sediado em Blumenau-SC

3. Autodoc, da CTE Inteligência, sediada em São Paulo-SP

4. Sudysoft, da JKMontagens, sediada em Trindade$\mathrm{GO}$

Tais ferramentas hoje possuem características tecnológicas mais avançadas envolvendo a computação em nuvem, uma tecnologia mais avançada do que aquela de Extranets apresentados nos trabalhos de pesquisa desenvolvidos no início da década.

\subsection{HISTÓRICO DA NORMA NBR ISO 9001:2008}

A norma ISO 9001 possui um histórico que acompanha o desenvolvimento dos conceitos da qualidade. Todos os seus conceitos sofreram ao longo do tempo diversas influências, passando de um enfoque na prevenção de defeitos (Versão de 1994) ao conceito atual de foco no cliente (Versão 2000), juntamente com uma visão sistêmica de processos.

A construção civil possui grande interesse na introdução da certificação da norma ISO 9001, pois 
segundo Lordêlo (2002), é a melhor maneira de se ter uma melhoria contínua dos processos e a consequente competitividade de seu produto no mercado.

Os itens da norma que exigem a aplicação de práticas de gestão para a certificação são: 4. Sistema de gestão da qualidade; 5. Responsabilidade da direção; 6. Gestão de recursos; 7. Realização do produto; 8 . Medição, análise e melhoria, cada qual com diversos subitens (ABNT, 2008).

A bibliografia em geral apresenta diversos estudos de caso a respeito da avaliação de empresas construtoras em relação à certificação da ISO 9001. Identificam-se alguns pontos em comum no que se refere às dificuldades encontradas na certificação. Entre elas podemos citar a dificuldade com gerenciamento da documentação necessária para certificação, falta de estratégia nos processos para que essa documentação auxilie na melhoria contínua dos processos e falta de engajamento da alta direção na implantação do Sistema de Gestão da Qualidade (OLIVEIRA, 2006; SEDDON, 2000).

\section{METODOLOGIA}

A forma de investigação do trabalho foi feita através de dois estudos de caso realizados com dois representantes de softwares de Gestão Operacional da Informação (GOI), como os citados anteriormente, porém com um enfoque na Gestão da Qualidade. A abordagem feita para a escolha dos softwares foi a de escolher duas aplicações totalmente díspares no sentido de relevância de mercado. O primeiro caso consistindo de uma aplicação com altos valores investidos, consequentemente uma lista de clientes com grande capacidade de investimento. Já o segundo caso, consistindo de uma aplicação com baixo valor investido e poucos usuários. Essa abordagem possibilitou traçar um amplo leque de possibilidades de investimento em inovação em um setor que possuem grande disparidade de capacidade de investimento e incentivo a inovação.

Para cada representante foram apresentados os itens da norma (Quadro 1) e através de entrevistas, foram levantadas questões correspondentes à TIC a partir das demonstração dos aplicativos (instalado em dispositivo móvel: tablet ou smartphone, ou não). Buscou-se mapear funcionalidades semelhantes existentes entre as ferramentas de TIC voltadas para gestão da qualidade e vantagens de uso quando aplicadas com dispositivos móveis para a implantação dos Sistemas de Gestão da Qualidade. Assim foi possível identificar se a ferramenta atendia primeiramente aos requisitos da norma e se tais funcionalidades possuíam mobilidade. Os resultados dessa análise foram apresentados em um segundo quadro (Quadro 2).

\section{ANÁLISE E INTERPRETAÇÃO DOS DADOS}

O primeiro software (Caso 1) analisado consiste em um grupo de aplicações que juntos compõem um pacote para a Gestão da Qualidade. A empresa desenvolvedora possui sede na cidade de São Paulo e possui uma infraestrutura de desenvolvimento , contando com equipes de desenvolvimento e uma carteira de clientes composta de grandes construtoras do mercado brasileiro. A ferramenta está ambientada na web, tendo a possibilidade de armazenamento de dados remoto (nuvem) oferecido pela empresa desenvolvedora ou armazenamento na própria sede da construtora, demandando uma infraestrutura de servidores para o sistema.

O segundo software (Caso 2) consistiu de um software desenvolvido e financiado por uma indústria metalúrgica do setor de alimentos no Estado de Goiás. Possui por objetivo, implantar, manter e disponibilizar on-line o Sistema da NBR ISO 9001:2008. O sistema tem apoio de um grupo de programadores gerenciados pelo Gerente da Qualidade da indústria metalúrgica e avaliado por um consultor de certificação da qualidade responsável pela certificação da indústria no ano de 2011.

O software mais abrangente nos requisitos da norma NBR ISO 9001:2008 e que atende melhor aos conceitos de mobilidade da informação é o Caso 1 estudado. Esta avaliação é embasada na tabulação dos dados representada no Quadro 2, em que para cada questionamento relacionado aos itens da norma ISO 9001 incluiu-se uma pontuação sobre a aplicabilidade do software avaliado. Também para cada questionamento avaliou-se se a funcionalidade correspondente no software possibilitava uma adaptação especifica para um dispositivo móvel.

Com esse método, constatou-se que no Caso 1 o aplicativo possuía um total de 18 de 23 itens da norma atendidos. Mostrando que as funcionalidades da ferramenta realmente estão coerentes com as determinações da norma ISO. Quanto a mobilidade, o Caso 1 demonstrou possuir 8 itens da norma sendo atendidos com a possibilidade de mobilidade do usuário. 
QUADRO 1: Itens da norma com questionamentos levantados.

\begin{tabular}{|c|c|c|}
\hline $\begin{array}{l}\text { Item da } \\
\text { Norma }\end{array}$ & Descrição & Questão levantada \\
\hline \multirow{2}{*}{4} & 4.1 Requisitos gerais & $\begin{array}{l}\text { O software disponibiliza os processos necessários para o Sistema de } \\
\text { Gestão da qualidade e sua aplicação? }\end{array}$ \\
\hline & 4.2 Requisitos de documentação & $\begin{array}{l}\text { O documento "Manual da Qualidade" está disponível para todos na } \\
\text { organização através do software? }\end{array}$ \\
\hline \multirow{6}{*}{5} & 5.1 Comprometimento da direção & $\begin{array}{l}\text { Existe algum campo para realização da analise crítica dos processo } \\
\text { que fique disponível para todos da organização? }\end{array}$ \\
\hline & 5.2 Foco no cliente & $\begin{array}{l}\text { Existe algum campo que disponibilize os requisitos do cliente para } \\
\text { todos da organização? }\end{array}$ \\
\hline & 5.3 Política da qualidade & $\begin{array}{l}\text { Possui algum campo que disponibiliza a Política da Qualidade para } \\
\text { todos da organização? }\end{array}$ \\
\hline & 5.4 Planejamento & $\begin{array}{l}\text { Existe algum campo que disponibilize controle de revisões de } \\
\text { processos e procedimentos? }\end{array}$ \\
\hline & $\begin{array}{l}\text { 5.5 Responsabilidade, autoridade e } \\
\text { comunicação }\end{array}$ & Fica clara a hierarquização dos usuários dentro do sistema? \\
\hline & $\begin{array}{l}\text { 5.6 Análise crítica pela } \\
\text { administração }\end{array}$ & $\begin{array}{l}\text { No caso positivo do item } 5.1 \text {, existe uma tela para documentação da } \\
\text { análise crítica? }\end{array}$ \\
\hline \multirow{4}{*}{6} & 6.1 Provisão de recursos & \\
\hline & 6.2 Recursos humanos & $\begin{array}{l}\text { Existe algum campo para preenchimento de treinamentos sobre } \\
\text { processos do sistema? }\end{array}$ \\
\hline & 6.3 Infraestrutura & \\
\hline & 6.4 Ambiente de trabalho & $\begin{array}{l}\text { Existe algum campo para preenchimento de conformidades do } \\
\text { ambiente de trabalho? }\end{array}$ \\
\hline \multirow{6}{*}{7} & $\begin{array}{l}\text { 7.1 Planejamento da realização do } \\
\text { produto }\end{array}$ & Existe algum campo que disponibilize o plano de qualidade da obra? \\
\hline & $\begin{array}{l}\text { 7.2 Processos relacionados a } \\
\text { clientes }\end{array}$ & $\begin{array}{l}\text { Existe algum campo para coleta de dados sobre o atendimento dos } \\
\text { requisitos do cliente na entrega ou pós-entrega? }\end{array}$ \\
\hline & 7.3 Projeto e desenvolvimento & Existe algum campo para a verificação e análise crítica de projetos? \\
\hline & 7.4 Aquisição & Existe algum campo para avaliação de fornecedores? \\
\hline & $\begin{array}{l}\text { 7.5 Produção e fornecimento de } \\
\text { serviço }\end{array}$ & $\begin{array}{l}\text { Existe algum campo que disponibilize os procedimentos executivos } \\
\text { de obras para todos os usuários ou parte deles? }\end{array}$ \\
\hline & $\begin{array}{l}\text { 7.6 Controle de dispositivos de } \\
\text { medição e monitoramento. }\end{array}$ & Existe algum campo para controle de equipamentos de medição? \\
\hline \multirow{5}{*}{8} & 8.1 Generalidades & \\
\hline & 8.2 Medição e monitoramento & Existe algum campo para preenchimento de pesquisas de satisfação? \\
\hline & $\begin{array}{l}\text { 8.3 Controle de produto não- } \\
\text { conforme }\end{array}$ & Existe algum campo para identificação do produto não-conforme? \\
\hline & 8.4 Análise de dados & $\begin{array}{l}\text { Existem relatórios suficientes abordando os conceitos de: satisfação } \\
\text { de clientes, conformidade com os requisitos do produto, ação } \\
\text { preventiva e fornecedores? }\end{array}$ \\
\hline & 8.5 Melhorias; & Existe alguma forma de alimentação de processos? \\
\hline
\end{tabular}


O Caso 2 demonstrou ser uma ferramenta pouco aplicada as determinações da norma, com apenas 10 itens sendo atendidos e nenhuma pontuação com uma ênfase móvel, por não possuir nenhuma funcionalidade adaptável a um dispositivo móvel. No Caso 2 o software, assim como o do Caso 1, esta ambientado on-line possibilitando suas funções de forma remota na leitura dos dados em um tablet dentro de um canteiro de obras. Porém, as suas aplicações ficam limitadas quando da inexistência de uma rede de dados de terceira geração (3G) ou uma rede Wireless de conexão com a internet.

Além desses fatores, foram identificadas incompatibilidades de determinadas funções da ferramenta com um browser de acesso remoto.

Outro fator limitante do software do Caso 2 é a incapacidade de preenchimento de determinados requisitos, como fichas de verificação de serviço e treinamentos de forma remota ou ainda a necessidade da impressão desses documentos em papel para validação dos mesmos no processo de auditoria. $O$ que se mostrou ineficiente na analise da aplicação da ferramenta em um canteiro de obra a partir de um diálogo com um Engenheiro Residente de uma empresa construtora que adotava o software do Caso 2 para o controle da Gestão da Qualidade.

\begin{tabular}{|c|c|c|c|c|c|}
\hline \multirow[b]{2}{*}{ Quant. } & \multirow[b]{2}{*}{ Item } & \multicolumn{2}{|c|}{ Caso 1} & \multicolumn{2}{|c|}{ Caso 2} \\
\hline & & $\begin{array}{c}\text { Resposta à } \\
\text { questão levantada }\end{array}$ & $\begin{array}{l}\text { Existe esta função } \\
\text { em aplicativo móvel }\end{array}$ & $\begin{array}{c}\text { Resposta à } \\
\text { questão levantada }\end{array}$ & $\begin{array}{l}\text { Existe esta função } \\
\text { em aplicativo móvel }\end{array}$ \\
\hline 1 & 4.1 & 1 & 1 & 1 & 0 \\
\hline 2 & 4.2 & 1 & 0 & 1 & 0 \\
\hline 3 & 5.1 & 1 & 0 & 1 & 0 \\
\hline 4 & 5.2 & 1 & 0 & 0 & 0 \\
\hline 5 & 5.3 & 1 & 0 & 1 & 0 \\
\hline 6 & 5.4 & 1 & 0 & 0 & 0 \\
\hline 7 & 5.5 & 1 & 1 & 1 & 0 \\
\hline 8 & 5.6 & 1 & 0 & 0 & 0 \\
\hline 9 & 6.1 & & & & \\
\hline 10 & 6.2 & 1 & 1 & 1 & 0 \\
\hline 11 & 6.3 & & & & \\
\hline 12 & 6.4 & 1 & 0 & 1 & 0 \\
\hline 13 & 7.1 & 1 & 1 & 1 & 0 \\
\hline 14 & 7.2 & 1 & 1 & 0 & 0 \\
\hline 15 & 7.3 & 1 & 0 & 0 & 0 \\
\hline 16 & 7.4 & 0 & 0 & 0 & 0 \\
\hline 17 & 7.5 & 1 & 1 & 1 & 0 \\
\hline 18 & 7.6 & 1 & 1 & 0 & 0 \\
\hline 19 & 8.1 & & & & \\
\hline 20 & 8.2 & 0 & 0 & 0 & 0 \\
\hline 21 & 8.3 & 1 & 1 & 1 & 0 \\
\hline 22 & 8.4 & 1 & 0 & 0 & 0 \\
\hline 23 & 8.5 & 1 & 0 & 0 & 0 \\
\hline \multirow[t]{2}{*}{ TOTAL } & 23 & 18 & 8 & 10 & 0 \\
\hline & $100 \%$ & $78 \%$ & $35 \%$ & $43 \%$ & $0 \%$ \\
\hline
\end{tabular}

Legenda 1 = Atendimento ao item da norma

$0=$ Não atendimento ao item da norma 


\section{CONSIDERAÇÕES FINAIS}

Ambos os casos estudados demonstraram que os softwares de Gestão Operacional da Informação (GOI), estão atendendo a um percentual moderado dos itens da Norma ISO. A mais adotada como critério de padronização e orientação a satisfação e melhoria contínua dos processos na construção civil.

O software do Caso 1 possuía hardware e software que possibilitavam ao usuário a entrada de dados sem conexão remota, porém com o carregamento dos dados em uma estação de trabalho conectados a internet e na sequencia a dispersão dessa informação via web. O Caso 2 demonstrou ser mais incipiente tecnologicamente, mas demostrou o interesse de desenvolvedores do setor em atender aos requisitos da norma e criar uma demanda de utilização das ferramentas de TIC na construção civil.

Com isso, pode-se considerar que aquelas dificuldades anteriormente citadas nos trabalhos de Nascimento e Santos (2002) e Souza e Amorin (2001) sobre a burocratização do fluxo da informação e dificuldades na implantação de inovações e melhoria dos processos inexistem atualmente no setor da construção. O setor que antes retratava dificuldades na comercialização e dispersão de tais tecnologias, atualmente se mostra aberto e demandando tais avanços, tendo em vista o número de softwares disponíveis no mercado e a competitividade atual do setor.

\section{REFERÊNCIAS BIBLIOGRÁFICAS}

ASSOCIAÇÃO BRASILEIRA DE NORMAS TÉCNICAS (ABNT). NBR ISO 9001:2008: Sistemas de gestão da qualidade Requisitos. Rio de Janeiro, 2008. 28p.

BOWDEN, S.; DORR, A.; THORPE, T.; ANUMBA, C. Mobile ICT support for construction process improvement. Automation in Construction, v. 15 , n. 5, p. 664-676. doi: 10.1016/j.autcon., 2006.

BRASIL. Lei no 9.472, de 16 de julho de 1997. Diário Oficial [da] República Federativa do Brasil, Poder Executivo, Brasília, DF, 16 dJUL. 1997.

BREGA, J. R. F.; LAURIS, J. R. P.; MOREIRA, P. R. C.; PEREIRA, R. C. FOB-USP Levantamento Epidemiológico em Saúde Bucal Utilizando Ferramentas Móveis, 2008.

CONCEIÇÃO, A. F. DA; PEREIRA, R. L.; REZENDE, J. V. P. et al. Projeto Borboleta: Ferramentas Móveis e Multimídia para Atenção Básica Domiciliar. . Recuperado de http://www.sbis.org.br/cbis11/arquivos/636.pdf, 2010.
CROCKER, P. A global mobile handset forecast: 2011-2015. GigaOM Pro!, p. 20. São Francisco. Recuperado maio 2, 2012, , 2011.

FÜHR, D. Análise e Modelagem de um sistema de CRM para Dispositivos Móveis. Novo Hamburgo: Feevale, 2007.

GARCIA, M. G. P. O financiamento à infraestrutura e a retomada do crescimento econômico sustentado. BNDES, Área de Planejamento, Departamento Econômico-DEPEC, 1995.

GIBBS, C. The rise of tablets in the enterprise. GigaOM Pro!, p. 19. Recuperado abril 2, 2012, , 2011.

GOMES, W. E. C. P. Plataforma de desenvolvimento de software baseado em componentes para dispositivos moveis. . Dissertação de Mestrado. Universidade Estadual de Campinas, 2005.

INSTITUTO DE PESQUISA ECONOMICA APLICADA - IPEA. DESAFIOS E OPORTUNIDADES DO SETOR DE TELECOMUNICAÇÕES NO BRASIL. , EixOS do desenvolvimento brasileiro. Brasília: Instituto de Pesquisa Economica Aplicada - IPEA. Recuperado de www.ipea.gov.br, 2010.

ISMAIL, S. M. Um ambiente virtual de aprendizagem que utiliza avaliação formativa, a tecnologia de mensagens curtas e dispositivos móveis. . Dissertação de Mestrado, Universidade Estadual de Campinas, 2011.

KOUTROUMPIS, P. The economic impact of broadband on growth: A simultaneous approach. Telecommunications Policy, v. 33, n. 9, p. 471-485, 2009.

LORDÊLO, P. M. Avaliação das modificações introduzidas pela versão 2000 da série de normas NBR ISO 9000 o caso das empresas construtoras de edifícios. São Paulo: EPUSP, 2002.

MIGLINSKI, C. A. Uma ferramenta para suporte ao desenvolvimento de software orientado a componente. . Dissertação de Mestrado, Universidade Estadual de Campinas, 2003.

DO NASCIMENTO, L. A.; SANTOS, E. T. Barreiras para o uso da tecnologia da informação na indústria da construção civil. II WORKSHOP NACIONAL - GESTÃO DO PROCESSO DE PROJETO NA CONSTRUÇÃO DE EDIFÍCIOS, 2002.

NEGÓCIOS EM EMPREENDIMENTOS. Mais inseridos do que nunca. , p. 132. Florianópolis , 2012, setembro.

OLIVEIRA, O. J. Gestão da qualidade: tópicos avançados. Cengage Learning Editores, 2006.

PEYERL, F. V. DESENVOLVIMENTO DE SISTEMA DE INFORMAÇÕES PARA CONTROLE DE CUSTOS DE OBRAS DE CONSTRUÇÃO CIVIL. Dissertação de Mestrado, UFPR, 2007. 
SCHAEFER, C. Protótipo de aplicativo para transmissão de dados a partir de dispositivos móveis aplicados a uma empresa de transporte. Trabalho de Conclusão de Curso, Universidade Regional de Blumenau, Blumenau, 2004.

SEDDON, J. The Case Against ISO 9000. Oak Tree Pr, 2000.

SIBALDO, M. A. A.; LOUREIRO, E.; BITTENCOURT, I. I.; COSTA, E. B. Infra-estrutura para Acesso a Comunidades Virtuais na Web Através de Dispositivos Móveis. Anais do Simpósio Brasileiro de Informática na Educação. Anais... v. 1, p.58-60, 2006.

SOUZA, J. M.; AMORIN, S. R. L. SIGMO-Sistema Integrado de Gerenciamento Móvel em Obras Coletânea Habitare-vol 2Inovação. Gestão da Qualidade e Produtividade e Disseminação do Conhecimento na Construção Habitacional, 2001.

VAVOULA, G. N.; LEFRERE, P.; O'MALLEY, C.; SHARPLES, M.; TAYLOR, J. Producing guidelines for learning, teaching and tutoring in a mobile environment. Wireless and Mobile Technologies in Education, 2004. Proceedings. The 2nd IEEE International Workshop on. Anais... p.173 - 176. doi: 10.1109/WMTE.2004.1281378, 2004.

WOLF, M. Enterprise mobility perceptions among IT decision makers. GigaOM Pro!, p. 13. São Francisco. Recuperado maio 2, 2012, , 2012.

ZEN, T. H. Diretrizes para o uso de tecnologia da informação no gerenciamento das informações do processo de planejamento e controle da produção. Dissertação de Mestrado, UFPR, 2006. 